\title{
Azathioprine Therapy in Multiple Sclerosis: Phosphoribosylated Metabolites and Thiopurine Methyltransferase Activity
}

\author{
Peter Albrecht ${ }^{1, *}$, Julia Tafazzoli-Lari ${ }^{1}$, Heiko Iven $^{2}$ and Andreas Moser ${ }^{1}$ \\ ${ }^{I}$ Neurochemical Research Group, Department of Neurology, University of Luebeck, Germany; ${ }^{2}$ Institute of Experimental \\ and Clinical Pharmacology and Toxicology, University of Luebeck, Germany
}

\begin{abstract}
Objective: In this prospective study, we examined the association between azathioprine dose, levels of its phosphoribosylated metabolites, and the activity of thiopurine methyltransferase in patients with multiple sclerosis (MS). Materials/Methods: Clinical data and blood samples were collected from 27 MS patients who were undergoing azathioprine treatment. In red blood cells, thiopurine methyltransferase (TPMT) activity was determined, and after hydrolysis and cleavage of the phosphoribosyl residue, amounts of 6-thioguanine (6-TG), 6-methyl-thioguanine (6-MTG), 6methylmercaptopurine (6-MMP) were measured. For clinical evaluation, the expanded disability status score (EDSS) and the multiple sclerosis functional composite (MSFC) were performed. Laboratory and clinical examinations were conducted twice with a 6-month-intervall. Results: Over a broad range of daily azathioprine dose, nearly constant levels of the immunosuppressive-active 6-TG (nucleotides) were found. There was, however, a marked relationship between daily azathioprine dose and 6-MMP nucleotide levels. Especially patients receiving an azathioprine dose of more than 1.5 $\mathrm{mg} / \mathrm{kg}$ per day in particular presented an exponential increase in 6-MMP levels when TPMT activity was higher than 45 $\mathrm{U} / \mathrm{g} \mathrm{Hb}$. All the biochemical measurements gave similar results when performed 6 months later. Conclusions: Patients with the combination of a high TPMT-activity and an azathioprine dose of more than $1.5 \mathrm{mg} / \mathrm{kg} / \mathrm{d}$ exhibit significantly increased 6-MMP nucleotide levels. These patients are thus at risk for hepatotoxic side effects. Determination of TPMT activity before azathioprine therapy and monitoring of its metabolites might provide guidance for dose individualization.
\end{abstract}

Keywords: Azathioprine, multiple sclerosis, phosphoribosylated metabolites, thiopurine methyltransferase activity.

\section{INTRODUCTION}

Azathioprine was introduced in MS therapy in the 1970s $[1,2]$. Since then, several studies have shown its effectiveness in the treatment of MS [3-5]. Additionally, it is used in the immunosuppressive treatment of other neurological autoimmune diseases, for example, myasthenia gravis [6,7]. Moreover, azathioprine is used widely in the treatment of other, non neurological, autoimmune and inflammatory conditions, for example rheumatoid arthritis (RA) or inflammatory bowel disease (IBD) $[8,9]$. It is licensed for MS therapy in Germany, and today, azathioprine represents an established second-line therapy [10]. However, azathioprine predisposes to adverse events. Apart from idiosyncratic side effects such as fever or gastroenterological disturbances, there are severe dose dependent side effects, in particular hepatoxicity and myelodepression. Azathioprine is a prodrug and metabolized via a biochemical sequence, which is summarized in (Fig. 1) [11]. The key-enzyme is thiopurine methyltransferse (EC: 2.1.1.67, TPMT) that exhibits an autosomal co-dominant polymorphism $[12,13]$. 6-Thioguanine nucleotides (6-TG nucleotides) are thought to be the immunosuppressive-active metabolites [14]. 6-TG nucleotides are incorporated into DNA, results in inhibition of lymphocyte proliferation. Different studies on thiopurines in IBD showed

*Address correspondence to this author at the Department of Neurology, University of Luebeck, Ratzeburger Allee 160, 23538 Luebeck, Germany; Tel: +49 451 5002930; Fax: +49 451 5002489;

E-mail: peter.albrecht@neuro.uni-luebeck.de that elevated levels of 6-TG nucleotides pose an increased risk of myelosuppression, whereas 6-methylmercaptopurine (6-MMP) nucleotides are related to an increased risk of hepatotoxicity [15]. As a result of the complex metabolism and genetic polymorphism of metabolic enzymes, there is wide interpatient variation in the concentrations of active and potentially toxic metabolites in patients under azathioprine therapy [16]. Despite these findings, traditional dosing strategies for azathioprine in MS therapy are based on weight or are empirically chosen. These strategies do not take into account the considerable interindividual differences in azathioprine metabolism. The objective of this study was to investigate levels of phosphoribosylated azathioprine metabolites as a function of daily dose and TPMT activity in MS patients.

\section{MATERIALS AND METHODS}

\section{Patients and Clinical Assessment}

This prospective study included 27 patients with definite MS (5 men, 22 women, mean age 49.1 years, range 27-70). Subjects were recruited from the Department of Neurology at the University of Luebeck, Germany. Further inclusion criteria were age of eighteen years or older and treatment with a constant daily dose of azathioprine for at least six months prior to the study. The study procedure included examination by trained neurologists using the extended disability status scale (EDSS) and the multiple sclerosis functional composite (MSFC) $[17,18]$. Blood samples were drawn at 
two points of time (T1 and T2) with an interval of about sixmonths. Other clinical data collected included course of MS, sex, age at time of diagnosis, dose of azathioprine, and body weight. The study was conducted according to the ethical standards laid down in the 1964 Declaration of Helsinki and was approved by the local ethics committee. Before participation, informed written consent was obtained from all participants.

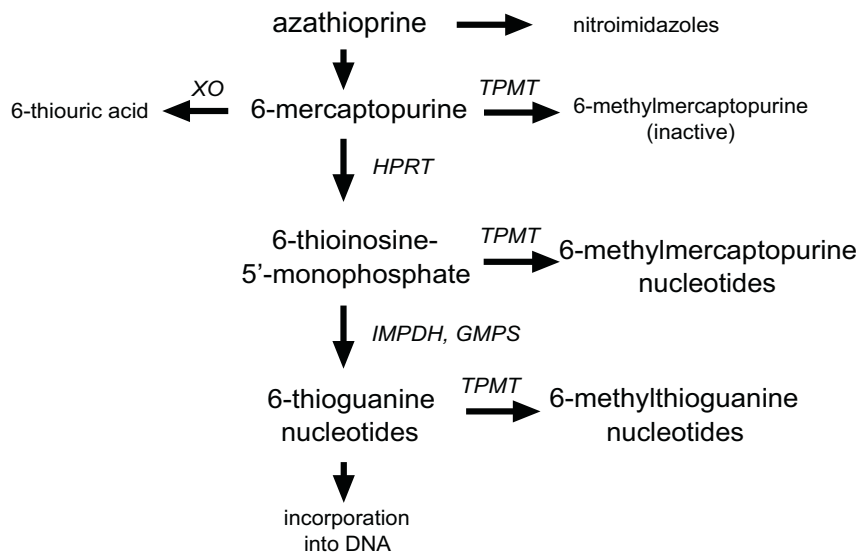

Fig. (1). Azathioprine metabolic pathway. Simplified azathioprine metabolism according to Karran \& Attard [11]. TPMT: Thiopurine methyltransferase. XO: Xanthine oxidase. HPRT: Hypoxanthine guanine phosphoribosyl transferase. IMPDH: Inosine monophosphate dehydrogenase. GMPS: Guanosine monophosphate synthase

\section{TPMT}

TPMT activity was determined using 6-thioguanine as substrate. We measured the formation of 6methylthioguanine with S-adenosyl-methionin as the methyl donor. Quantitative measurement of 6-methylthioguanine was performed with HPLC and fluorescence detection. One unit of enzyme activity represents the formation of $1 \mathrm{nmol}$ of 6-methylthioguanine per hour [19].

\section{Azathioprine Metabolites}

Amounts of 6-methylmercaptopurine (6-MMP), 6thioguanine (6-TG), and 6-methylthioguanine (6-MTG) were measured after hydrolysis and cleavage from the phosphoribosylated residue in red blood cells (RBC) by HPLC techniques [20]. Assay precision was measured using internal quality controls to ensure validity and reliability.

\section{Statistics}

Statistical analysis was performed using SPSS. Descriptive analysis included frequency, mean, standard error of mean, standard deviation of mean, median, minimum and maximum. Medians were given for better comparability with the clinical scores. Different statistical methods (ANOVA, and post hoc t-test for unpaired samples) were used when appropriate, logistic regression was used to adjust for potential cofounders. $\mathrm{P}$ was significant at 0.05 .

\section{RESULTS}

\section{Study Group Patients}

A total of $27 \mathrm{MS}$ patients were recruited for the study. All patients were on azathioprine treatment for at least 6 months before participating in the study. Patient details are summarized in (Table 1). There was no overall significant change of EDSS or MSFC over the period of time between the first and second visit (Table 2). Also, TPMT activity and the concentrations of the metabolites did not change between T1 and T2 (Table 2).

Table 1. Characteristics of the 27 multiple sclerosis patients treated with azathioprine at 1 st visit. *Values are median (range).

\begin{tabular}{|c|c|}
\hline Sex (F:M) & $\mathbf{2 2 : 5}$ \\
\hline \hline Relapsing Remitting & 16 \\
\hline $\begin{array}{c}\text { Relapsing Remitting secondary } \\
\text { progressive }\end{array}$ & 9 \\
\hline Primary Progressive & 2 \\
\hline Age (years) & $49(27-70$ \\
\hline Duration of MS (years) & $14(6-40)$ \\
\hline Age at onset of MS (years) & $34(21-49)$ \\
\hline weight $(\mathrm{kg}) *$ & $66(47-107$ \\
\hline BMI (kg/m $\left.{ }^{2}\right)^{*}$ & $24.1(16.7-32)$ \\
\hline Azathioprine dose (mg/day) & $100(50-200)$ \\
\hline Azathioprine dose (mg/kg/day)* & $1.7(0.5-2.7)$ \\
\hline
\end{tabular}

\section{TPMT}

TPMT activity was measured twice, with an approximate time interval of six months. Values showed a Gaussian distribution. Mean enzyme activity was slightly higher after six months (T1 mean $34.4 \pm 1.7$; T2 mean $41.9 \pm 1.34$ ), however without significant difference $(\mathrm{p}=0.202$; Table 2$)$. All patients were in the homozygous high range $(>14 \mathrm{U} / \mathrm{g} \mathrm{Hb})$. TPMT activity did not correlate with patients' age or disease duration (data not shown). Enzyme induction by azathioprine could not be observed since TPMT activity was not related to azathioprine dose. ANOVA gave $\mathrm{R}^{2}$ value of 0.077 which means that the azathioprine dose accounts for only $7.7 \%$ of the activity variance.

\section{Azathioprine Metabolites and Dose}

Amounts of phosphoribosylated azathioprine metabolites (nucleotides) were quantified after acid hydrolysis. 6Thioguanine (6-TG) was measured twice in red blood cells (ng/g Hb) (Table 2). Initial median [range] erythrocyte 6-TG levels (415, [192 -1174]) did not significantly change during the months of treatment $(461,[132-1749])$. 6-TG showed only a slight tendency, but not significant enhancement when daily azathioprine dose increased for patients, with spearman coefficient of $r=0.087$ ( $\mathrm{p}=0.678$ ) (Fig. 2a).

Levels of 6-methylthioguanine (6-MTG) clearly increased with daily azathioprine dose at $\mathrm{T} 1$. The relationship appeared linear and was statistically significant with spearman coefficient of $r=0.47(p=0.018)$. ANOVA also showed significant variance of regression $(\mathrm{p}=0.006)$ (Fig. 2b). 
Table 2. Clinical scores, TPMT activity and azathioprine metabolites of patients at T1 and T2.

\begin{tabular}{|c|c|c|c|}
\hline Parameter & T1 (1 ${ }^{\text {st }}$ Visit) & T2 $\left(2^{\text {nd }}\right.$ Visit $)$ & P value \\
\hline \hline EDSS (points) median (range) & $2.5(1-7)$ & $2.5(0-7)$ & 0.737 \\
\hline MSFC (Z-Score) median (range) & $-0.07(-0.92-4.64)$ & $-0.04(-0.71-0.71)$ & 0.559 \\
\hline TPMT activity median (range), U/g Hb & $43(21-57)$ & $42(23-54)$ & 0.202 \\
\hline 6-TG median (range), ng/g Hb & $415(192-1174)$ & $461(132-1749)$ & 0.178 \\
\hline 6-MTG median (range), ng/g Hb & $411(45-896)$ & $389(62-3165)$ & 0.615 \\
\hline 6-MMP median (range), ng/g Hb & $9407(904-59149)$ & $7423(408-37098)$ & 0.101 \\
\hline
\end{tabular}

Taken from all patients, marked enhancement of 6methylmercaptopurine (6-MMP) levels was observed when high doses of azathioprine were given. In contrast to 6MTG, the relationship appeared as an exponential growth (ANOVA $\mathrm{p}=0.001$; Fig. 2c).

\section{Effect of Azathioprine Dose and TPMT Activity on Nu- cleotide Levels}

To evaluate combined effects of azathioprine dose and TMPT activity on nucleotide levels, patients were divided into four subgroups: patients with low TPMT activity and low azathioprine dose (group $A, n=6$ ), patients with high TPMT activity and low dose (group $\mathrm{B}, \mathrm{n}=3$ ), patients with low TMPT activity and high dose (group $\mathrm{C} ; \mathrm{n}=12$ ) and patients with high TMPT activity and high dose (group D, $\mathrm{n}=6$ ). Since the median of TPMT activity of all patients was $43 \mathrm{U} / \mathrm{g} \mathrm{Hb}$, we set the limiting value for TPMT activity as 45 $\mathrm{U} / \mathrm{g} \mathrm{Hb}$. Limiting value for the dose was $1.5 \mathrm{mg} / \mathrm{kg}$ per day. As shown in (Table 3), there were no statistically significant differences in 6-TG or 6-MTG nucleotide levels between groups. Lowest 6-MMP nucleotide levels were found in group A. In group B, amounts of phosphoribosylated 6MMP were moderately enhanced. In group $\mathrm{C}$ patients (low TPMT activity but high azathioprine dose of more than 1.5 $\mathrm{mg} / \mathrm{kg}$ per day), 6-MMP levels were three times higher compared to group A. However, highest 6-MMP levels could be observed in group D (patients with high TMPT activity and high dose). Here, levels were approximately 12 times higher than in group A patients. Fig. (2d) illustrates these findings with different exponential growth curves according to high or low TMPT activity.

\section{DISCUSSION}

To our knowledge, this is the first study that presents azathioprine data to metabolites and thiopurine methyltransferase (TPMT) activity in MS patients who are on long term treatment. Our results showed marked relationship between daily azathioprine dose and levels of 6methylmercaptopurine (6-MMP) nucleotides in MS patients predominantly with TPMT-activity higher than $45 \mathrm{U} / \mathrm{g} \mathrm{Hb}$. Most studies concerning the use of 6-mercaptopurine (6-MP) of which azathioprine is the prodrug, and measurement of the metabolites that have been conducted in patients with inflammatory bowel disease (IBD) [21, 22]. It has been noted that some patients with IBD did not respond to an immunosuppressive therapy with azathioprine. Dubinsky et al. could demonstrate that these patients did not exhibit significant increase of the immunosuppressive active metabolite 6thioguanine (6-TG) nucleotide despite escalation of azathioprine doses. Since, in contrast to 6-TG nucleotides, amounts of phosphoribosylated 6-MMP significantly increased, they concluded that some IBD patients preferentially metabolize 6-MP to 6-MMP rather than to 6-TG nucleotides [23]. Our data may confirm these findings also for MS patients. According to our data, this subgroup consisted of patients with normal to high TPMT activity and also high azathioprine dose treatment. We suppose that in those patients high enzyme activity "shunts" the azathioprine metabolism to 6MMP nucleotides. Further increase of a daily azathioprine dose would lead to an enhancement of 6-MMP nucleotides, however, without significant increase of 6-TG nucleotides. Here, TPMT may stabilize levels of 6-TG nucleotides over a broad range of azathioprine doses by methylation to phosphoribosylated 6-MMP and 6-MTG (see Fig. 1). Therefore, two important points can be derived from this fact for clinicians. If patients with high TPMT activity (probably higher than $45 \mathrm{U} / \mathrm{g} \mathrm{Hb}$ ) fail to respond to azathioprine treatment, especially at high doses (e.g. $1.5 \mathrm{mg} / \mathrm{kg} / \mathrm{d}$ ), further escalation would probably not lead to improvement. In this case, however, the risk of hepatotoxic side effects increases since the phosphoribosylated metabolite 6-MMP is clearly associated with hepatotoxicity [24]. Considering that on one hand 6-TG nucleotides are thought to be the predominant immunosuppressive active metabolites of thiopurines and on the other hand elevated 6-MMP nucleotides may result in hepatotoxic effects, we propose a maximum daily azathioprine dose of $1.5 \mathrm{mg} / \mathrm{kg}$. In this context, Asseldonk et al. described IBD patients who had experienced hepatotoxicity due to 6MMP nucleotides by treatment with azathioprine or 6mercaptopurine. After switching the therapy to 6thioguanine, immunosuppressive substances enter the metabolic pathway "further down", and a "shunting away" to 6MMP nucleotides by TMPT is avoided. This strategy resulted in a reduced hepatotoxicity rate [25]. On the other hand, prior to any azathioprine treatment enzyme activity of TPMT should also be detected since patients with reduced enzyme activity exist, e.g. with other than homozygotic TMPT gene background [26]. Patients with a low TMPT activity were prone to develop myelotoxic side effects due to immunosuppressive active 6-TG nucleotides, while amounts of 6-MMP nucleotides were rather low [24, 27]. Since in our study enzyme activities ranged from normal to high values, these data can not support findings related to 6-TG 

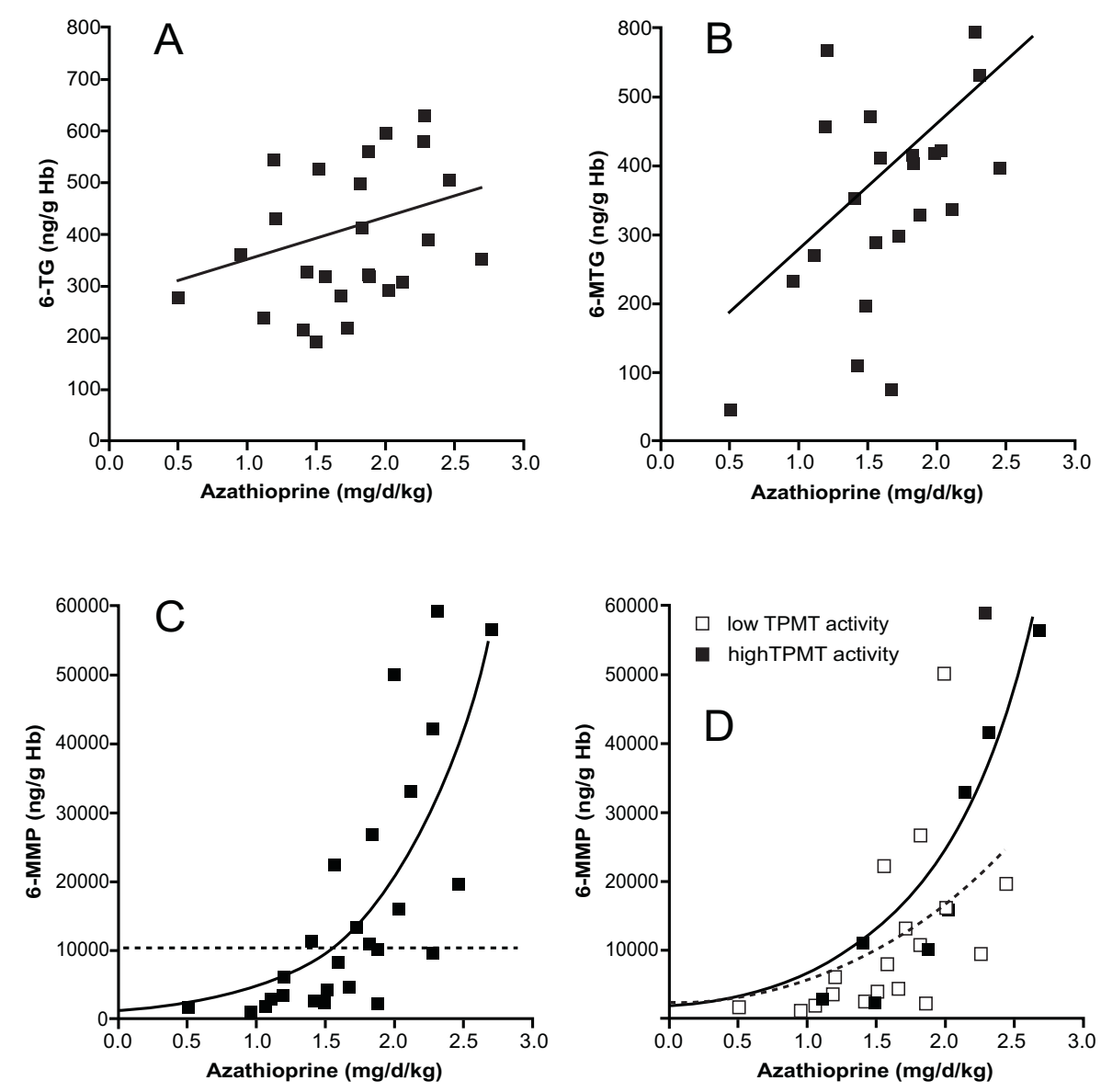

Fig. (2). Relationship between daily azathioprine dose and nucleotide levels of 6-thioguanine (6-TG; A), 6-methylthioguanine (6-MTG; B), and 6-methylmercaptopurine (6-MMP; C and D). Compounds were detected by HPLC technique after hydrolysis of the phosphoribosylated nucleotides. High TPMT activity was defined as enzyme activity of more than $45 \mathrm{U} / \mathrm{g} \mathrm{Hb}$ (D).

Table 3. Azathioprine metabolite levels in patients with low TPMT and low dose (group A, n=6), patients with high TPMT activity and low dose (Group B, $n=3$ ), patients with low TMPT activity and high dose (Group C; $n=12$ ) and patients with high TMPT activity and high dose (Group $D, n=6)$. Limiting value for daily azathioprine dose: $1.5 \mathrm{mg} / \mathrm{kg}$, limiting value for TPMT activity: $45 \mathrm{U} / \mathrm{g}$ Hb. ${ }^{*}$ p $<0.05$ significant, when compared to Group $A .{ }^{* *}<0.05$ significant, when compared to Group C.

\begin{tabular}{|c|c|c|c|}
\hline & $\begin{array}{c}\text { 6-TG } \\
(\mathbf{n g} / \mathbf{g H b})\end{array}$ & $\begin{array}{c}\text { 6-MTG } \\
\mathbf{( n g / g H b})\end{array}$ & $\begin{array}{c}\text { 6-MMP } \\
(\mathbf{n g} / \mathbf{g H b})\end{array}$ \\
\hline $\begin{array}{c}\text { Group A (low TPMT, low dose; } n=6) \\
\text { mean } \pm \text { SD (min.-max.) }\end{array}$ & $518 \pm 136(279-1174)$ & $346 \pm 103(45-664)$ & $2835 \pm 747(1049-6086)$ \\
\hline $\begin{array}{c}\text { Group B (high TPMT, low dose; } n=3) \\
\text { mean } \pm \text { SD (min.-max.) }\end{array}$ & $215 \pm 13(192-240)$ & $273 \pm 44(197-352)$ & $5482 \pm 2745(2494-10966)$ \\
\hline $\begin{array}{c}\text { Group C (low TPMT, high dose; } n=12) \\
\text { mean } \pm \text { SD (min.-max.) }\end{array}$ & $480 \pm 53(221-852)$ & $405 \pm 42(75-669)$ & $15491 \pm 3875(904-50236)^{*}$ \\
\hline $\begin{array}{c}\text { Group D (high TPMT, high dose; } n=6) \\
\text { mean } \pm \text { SD (min.-max.) }\end{array}$ & $427 \pm 55(308-630)$ & $569 \pm 90(330-896)$ & $33886 \pm 9622(2297-59149)^{* *}$ \\
\hline
\end{tabular}

nucleotides in the case of low TPMT activity, e.g. lower than $14 \mathrm{U} / \mathrm{g} \mathrm{Hb}$.

Our study has, however, additional limitations. The MS patient group is rather small and heterogenous. These factors cause statistical restrictions especially regarding the interpretation of the clinical data. Subsequently, the information our clinical data can give, is the fact that the group represents a typical sample of MS patients. It is not possible to link clinical data in any way to azathioprine dose, TPMT activity or the metabolites levels. In particular it is not possible to draw any conclusions concerning the impact of these factors on the clinical response (or the lack of it). The patients' azathio- 
prine doses were indeed not standardized. Yet, the data described above led us to suggest that after a 6 month stabilization period of individual treatment, TPMT activity as well as azathioprine metabolite levels are rather stable over time. Most findings regarding azathioprine therapy and analysis of metabolites were gained in studies on IBD or other internal diseases but not on Multiple Sclerosis. It is unclear, to which extent this knowledge can be transferred to the treatment of MS patients.

\section{CONCLUSION}

We could observe a strong relationship between daily azathioprine dose and hepatotoxic side-effect-inducing 6MMP nucleotides in MS patients with high TPMT activity. Thus, we would propose measurement of TPMT activity before and phosphoribosylated metabolites measurements during azathioprine treatment in order to reduce toxic side effects. Further studies are needed to optimize azathioprine therapy and to evaluate the clinical impact of this compound on Multiple Sclerosis. Additionally, with teriflunomide inhibiting dihydroorotate dehydrogenase (EC 1.3.3.1) and pyrimidine biosynthesis, the therapeutical approach of DNA subunit modification is now renewed in treatment of Multiple Sclerosis.

\section{CONFLICT OF INTEREST}

The authors confirm that this article content has no conflicts of interest.

\section{ACKNOWLEDGEMENTS}

This study was supported in part by a grant of Glaxo SmithKline, Germany.

\section{PATIENT'S CONSENT}

Declared none.

\section{REFERENCES}

[1] Müller J, Kreiner R, Sauermann W. Preliminary results of imurek treatment of multiple sclerosis. Psychiatr Neurol Med Psychol 1976; 28: 755-61.

[2] Abdou NI, Zweiman B, Casella SR. Effects of azathioprine therapy on bone marrow-dependent and thymus-dependent cells in man. Clin Exp Immunol 1973; 13: 55-64.

[3] Markovic-Plese S, Bielekova B, Kadom N, et al. Longitudinal MRI Study. The effects of azathioprine in MS patients refractory to interferon $\beta-1 b$. Neurology 2003; 60: 1849-51.

[4] Massacesi L, Parigi A, Barilaro A, et al. Efficacy of Azathioprine on multiple sclerosis new brain lesions evaluated using magnetic resonance imaging. Arch Neurol 2005; 62: 1843-7.

[5] Casetta I, Iuliano G, Filippini G. Azathioprine for multiple sclerosis. Cochrane Database Syst Rev 2007; 17: CD003982.

[6] Gilhus NE, Owe JF, Hoff JM, Romi F, Skeie GO, Aarli JA. Myasthenia gravis: a review of available treatment approaches. Autoimmune Dis 2011; 2011: 847393.

[7] Sanders DB, Evoli A. Immunosuppressive therapies in myasthenia gravis. Autoimmunity 2010; 43: 428-35.

[8] Pearson DC, May GR, Fick GH, Sutherland LR Azathioprine and 6-mercaptopurine in Crohn disease. A meta-analysis. Ann Intern Med 1995; 123: 132-42.
[9] Suarez-Almazor ME, Spooner C, Belseck E. Azathioprine for treating rheumatoid arthritis. Cochrane Database Syst Rev 2000; 4: CD001461.

[10] Kieseier BC, Jeffery DR. Chemotherapeutics in the treatment of multiple sclerosis. Ther Adv Neurol Disord 2010; 3: 277-91.

[11] Karran P, Attard K. Thiopurines in current medical practice: molecular mechanisms and contributions to therapy-related cancer. Nat Rev Cancer 2008; 8: 24-36.

[12] Krynetski EY, Evans WE. Genetic polymorphism of thiopurine Smethyltransferase: molecular mechanisms and clinical importance. Pharmacology 2000; 61: 136-46.

[13] Schwab M, Schäffeler E, Marx C, et al. Azathioprine therapy and adverse drug reactions in patients with inflammatory bowel disease: impact of thiopurine S-methyltransferase polymorphism. Pharmacogenetics 2002; 12: 429-36.

[14] Coulthard S, Hogarth L. The thiopurines: an update. Investigational New Drugs 2005; 23: 523-32.

[15] Dubinsky MC. Azathioprine, 6-mercaptopurine in inflammatory bowel disease: pharmacology, efficacy, and safety. Clin Gastroenterol Hepatol 2004; 2: 731-43.

[16] Hindorf U, Lindquist M, Peterson C, et al. Pharmacogenetics during standardised initiation of thiopurine treatement in inflammatory bowel disease. Gut 2006; 55:1423-31.

[17] Kurtzke JF. Rating neurologic impairment in multiple sclerosis: an expanded disability status scale (EDSS). Neurology 1983; 33: 1444-52.

[18] Fischer JS, Rudick RA, Cutter GR, Reingold SC. The Multiple Sclerosis Functional Composite Measure (MSFC): an integrated approach to MS clinical outcome assessment. National MS Society Clinical Outcome Assessment Task Force. Mult Scler 1999; 5: 24450 .

[19] Kröplin T, Weyer N, Gutsche S, Iven H. Thiopurine Smethyltransferase activity in human erythrocytes: a new HPLC method using 6-thioguanine as substrate. Eur J Clin Pharmacol 1998; 54: 263-9.

[20] Erdmann GR, France LA, Bostrom BC, Canafax DM. A reversed phase high performance liquid chromatography approach in determining total red blood cell concentrations of 6-thioguanine, 6 mercaptopurine, methylthioguanine, and methylmercaptopurine in a patient receiving thiopurine therapy. Biomed Chromatogr 1990; 4: 47-51

[21] Cuffari C, Hunt S, Bayless T. Utilisation of erythrocyte 6thioguanine metabolite levels to optimise azathioprine therapy in patients with inflammatory bowel disease. Gut 2001; 48: 642-6.

[22] Gearry RB, Barclay ML. Azathioprine and 6-mercaptopurine pharmacogenetics and metabolite monitoring in inflammatory bowel disease. J Gastroenterol Hepatol 2005; 20: 1149-57.

[23] Dubinsky MC, Yang H, Hassard PV, et al. 6-MP metabolite profiles provide a biochemical explanation for 6-MP resistance in patients with inflammatory bowel disease. Gastroenterology 2002; 122: $904-15$

[24] Bradford K, Shih DQ. Optimizing 6-mercaptopurine and azathioprine therapy in the management of inflammatory bowel disease. World J Gastroenterol 2011; 17: 4166-73.

[25] van Asseldonk DP, Seinen ML, de Boer NK, van Bodegraven AA, Mulder CJ. Hepatotoxicity associated with 6-methyl mercaptopurine formation during azathioprine and 6-mercaptopurine therapy does not occur on the short-term during 6-thioguanine therapy in IBD treatment. J Crohns Colitis 2012; 6: 95-101.

[26] Teml A, Schaeffeler E, Herrlinger KR, Klotz U, Schwab M. Thiopurine treatment in inflammatory bowel disease: clinical pharmacology and implication of pharmacogenetically guided dosing. Clin Pharmacokinet 2007; 46: 187-208.

[27] Gisbert JP, Niño P, Rodrigo L, Cara C, Guijarro LG. Thiopurine methyltransferase (TPMT) activity and adverse effects of azathioprine in inflammatory bowel disease: long-term follow-up study of 394 patients. Am J Gastroenterol 2006; 101: 2769-76. 